\title{
Quantum phase transitions into Kondo states in bilayer graphene
}

\author{
Diego Mastrogiuseppe, ${ }^{1,2}$ Arturo Wong, ${ }^{3}$ Kevin Ingersent, ${ }^{3}$ Sergio E. Ulloa, ${ }^{1,2}$ and Nancy Sandler ${ }^{1,2}$ \\ ${ }^{1}$ Department of Physics and Astronomy, and Nanoscale and Quantum Phenomena Institute, Ohio University, Athens, Ohio 45701-2979, USA \\ ${ }^{2}$ Dahlem Center for Complex Quantum Systems and Fachbereich Physik, Freie Universität Berlin, 14195 Berlin, Germany \\ ${ }^{3}$ Department of Physics, University of Florida, P.O. Box 118440, Gainesville, Florida 32611-8440, USA \\ (Received 1 October 2013; revised manuscript received 22 January 2014; published 3 February 2014)
}

\begin{abstract}
We study a magnetic impurity intercalated in bilayer graphene. A representative configuration generates a hybridization function with strong dependence on the conduction-electron energy, including a full gap with one hard and one soft edge. Shifts of the chemical potential via gating or doping drive the system between non-Kondo (free-moment) and Kondo-screened phases, with strong variation of the Kondo scale. Quantum phase transitions near the soft edge are of Kosterlitz-Thouless type, while others are first order. Near the hard edge, a bound-state singlet appears inside the gap; although of single-particle character, its signatures in scanning tunneling spectroscopy are very similar to those arising from a many-body Kondo resonance.
\end{abstract}

One of the remarkable manifestations of cooperative phenomena in condensed matter is the many-body screening of a magnetic impurity in a nonmagnetic metal. The Kondo effect, well understood in ordinary metals [1], acquires added complexity in cases where the host density of states (DOS) varies strongly with energy $E$ near the chemical potential $\mu$. The pseudogap Kondo problem [2-6] with a DOS $\rho(E) \propto$ $|E-\mu|^{r}$ (realized, for example, for $r=1$ in strongly correlated systems such as high-temperature superconductors [7]) exhibits a rich phase diagram that depends on the band exponent $r$, the impurity-host exchange coupling $J$, and the presence or absence of particle-hole (p-h) symmetry.

Similar DOS features appear in layered low-dimensional materials such as graphene and transition metal dichalcogenides $[8,9]$. In particular, the technologically and conceptually important issue of creating local magnetic moments in graphene has been the focus of many recent theoretical [10-21] and experimental [22-25] studies, with controversial results (see [26] for an overview).

An even more complex DOS appears in bilayer graphene (BLG), a material that can be gapped by gating, and thus has attracted much attention for possible device applications [27]. The variety of microscopic environments for impurities combined with an easy tunability makes BLG highly promising for the study of quantum phase transitions (QPTs) into various Kondo states [28,29]. Thus, BLG appears to be a natural prototype for Kondo physics in layered materials.

This Rapid Communication explores impurity QPTs for a representative configuration of an intercalated spin $\sigma=$ $1 / 2$ magnetic impurity in BLG. This setup is described by an Anderson impurity model with an energy-dependent hybridization featuring a gap that has one hard and one soft edge. Under variation of the chemical potential $\mu$, the system passes from a free-moment (FM) phase to a Kondo phase featuring a strong $\mu$ dependence of the Kondo temperature scale. The QPTs found near the soft hybridization edge are of Kosterlitz-Thouless type, while all other QPTs are first order. We present thermodynamic and spectral properties near these QPTs, and discuss some of their consequences for spectroscopy measurements. For $\mu$ near the hard hybridization edge, the FM phase exhibits a singlet bound state inside the gap. This bound state is of single-particle character, but may have signatures in scanning tunneling spectroscopy very similar to those arising from a many-body Kondo resonance. Many of these features derive from the nature of the underlying DOS and are shared by other impurity configurations. More importantly, as the chemical potential can be effectively shifted by symmetric gating or homogeneous doping of the sample, the predicted properties should be accessible in available experimental setups, enabling detailed exploration of the physics of QPTs.

Bernal-stacked BLG [see Fig. 1(a)] is modeled with a realspace tight-binding Hamiltonian [30] with nearest-neighbor intralayer hopping amplitude $t$ and interlayer hopping $t_{\perp} \simeq$ $0.1 t$ between A-sublattice sites in layer 1 and $\mathrm{B}$-sublattice sites in layer 2. After Fourier transformation, this Hamiltonian can be expanded around inequivalent Brillouin zone corners $K_{ \pm}$that form the centers of two valleys $\tau= \pm$ in the gapless spectrum of neutral BLG. Diagonalization of the $4 \times 4$ Hamiltonian describing each valley [30,31] yields hyperbolic bands labeled $\alpha, \alpha^{\prime}= \pm$ [see Fig. 1(b)] having dispersion $E_{\alpha, \alpha^{\prime}}(q)=\alpha t_{\perp} / 2+\alpha^{\prime} \sqrt{\left(v_{F} q\right)^{2}+\left(t_{\perp} / 2\right)^{2}}$, where $q$ is the magnitude of the wave vector $\mathbf{q}$ measured relative to the zone corner and $v_{F}=3 a t / 2$ is the Fermi velocity of graphene. The combined DOS of both valleys (per spin orientation, per unit cell of area $\Omega_{0}$ ),

$$
\rho(E)=\frac{\Omega_{0}}{\pi v_{F}^{2}}\left[|E|+\frac{t_{\perp}}{2}+\Theta\left(|E|-t_{\perp}\right)\left(|E|-\frac{t_{\perp}}{2}\right)\right],
$$

has jumps at $E= \pm t_{\perp}$ [dashed line in Fig. 1(c)]. When its layers are gated symmetrically to yield a common chemical potential $\mu$ or homogeneously doped via intercalates, BLG is described by an eight-band low-energy effective Hamiltonian (per spin) $H_{\mathrm{BLG}}=\sum_{\alpha, \alpha^{\prime}, \tau, \sigma, \mathbf{q}}\left[E_{\alpha, \alpha^{\prime}}(q)-\right.$ $\mu] c_{\alpha, \alpha^{\prime}, \tau, \sigma}^{\dagger}(\mathbf{q}) c_{\alpha, \alpha^{\prime}, \tau, \sigma}(\mathbf{q})$. We take $t=3.0 \mathrm{eV}, t_{\perp}=0.3 \mathrm{eV}$, and $a=1.42 \AA$, in which case $v_{F} \simeq 10^{6} \mathrm{~m} / \mathrm{s}$ and $H_{\mathrm{BLG}}$ has an effective half-bandwidth $D \simeq 2.5 \mathrm{eV}$, which we take as the energy unit in our calculations.

An intercalated impurity can occupy one of several inequivalent positions [32]. We focus on the highsymmetry configuration shown in Fig. 1(b), described by an Anderson Hamiltonian $\bar{H}_{A}=H_{\mathrm{BLG}}+\epsilon_{d} n_{d}+U n_{d \uparrow} n_{d \downarrow}+$ $N_{c}^{-1 / 2} \sum_{\sigma}\left\{\left[V_{1} a_{1, \sigma}^{\dagger}(\mathbf{0})+V_{2} b_{2, \sigma}^{\dagger}(\mathbf{0})\right] d_{\sigma}+\right.$ H.c. $\}$, where $n_{d}=$ 

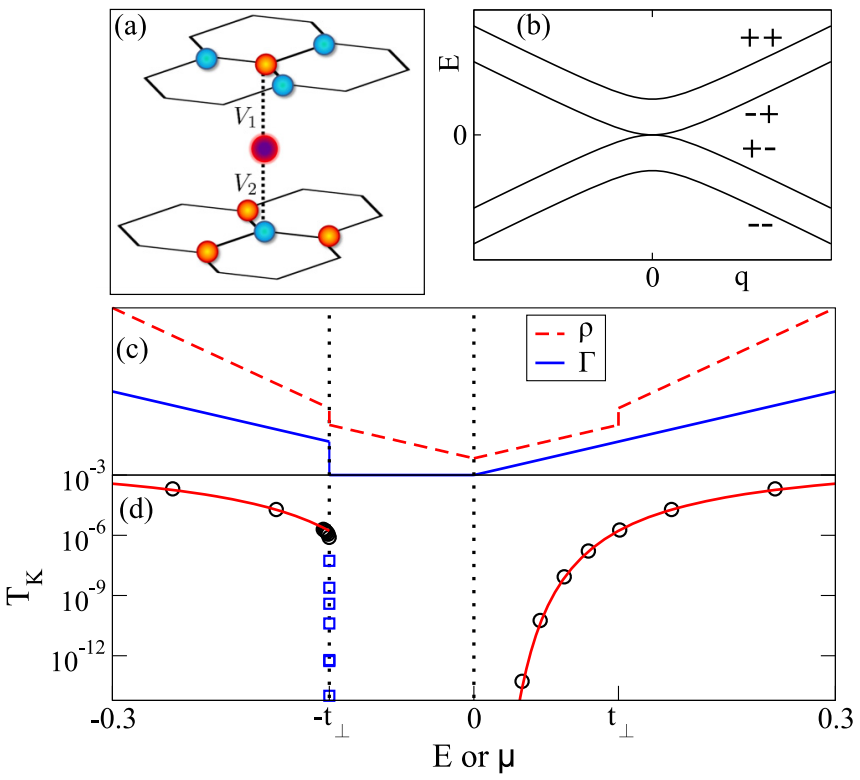

FIG. 1. (Color online) (a) Lattice structure of Bernal-stacked BLG, showing impurity configuration considered here. (b) Dispersion $E_{\alpha \alpha^{\prime}}(q)$ of the four bands along a line through one of the Brillouinzone corners $K_{ \pm}$(where $q=0$ ). (c) BLG density of states $\rho(E)$ (dashed line) and hybridization function $\Gamma(E)$ for the impurity configuration in (a) with $V_{1}=V_{2}$ (solid line). (d) Kondo temperature $T_{K}$ for $U=-2 \epsilon_{d}=0.1$ and $V_{1}=V_{2}=0.21$ vs chemical potential $\mu$ outside (circles) and inside (squares) the hybridization gap. The (red) line shows the result of substituting $\Gamma(\mu)$ into Haldane's formula for $T_{K}$.

$n_{d \uparrow}+n_{d \downarrow}$ with $n_{d \sigma}=d_{\sigma}^{\dagger} d_{\sigma}$ being the impurity number operator for spin $\sigma= \pm 1 / 2, \epsilon_{d}$ is the impurity level energy relative to the chemical potential, $U$ is the local Coulomb repulsion, and $N_{c}$ is the number of BLG unit cells. The last term in $\bar{H}_{A}$ describes tunneling of an electron between the impurity and the nearest sublattice-A atom in layer 1 or the nearest B-sublattice atom in layer 2 . Transformation to the eigenbasis of $H_{\mathrm{BLG}}$ and thence to an energy representation yields [31]

$$
\begin{aligned}
H_{A}= & \sum_{\sigma} \int_{-D}^{D} d E(E-\mu) c_{E \sigma}^{\dagger} c_{E \sigma}+\epsilon_{d} n_{d}+U n_{d \uparrow} n_{d \downarrow} \\
& +\sum_{\sigma} \int_{-D}^{D} d E \sqrt{2 \Gamma(E) / \pi}\left(c_{E \sigma}^{\dagger} d_{\sigma}+\text { H.c. }\right) .
\end{aligned}
$$

Here $c_{E \sigma}^{\dagger}$, satisfying $\left\{c_{E \sigma}^{\dagger}, c_{E^{\prime} \sigma^{\prime}}\right\}=\delta\left(E-E^{\prime}\right) \delta_{\sigma, \sigma^{\prime}}$, creates an electron in the single linear combination of band states of energy $E$ that hybridizes with the impurity, and we have dropped contributions from all the other (decoupled) linear combinations of band states. $H_{A}$ represents a conventional Anderson model apart from the unusual energy dependence of the hybridization function

$$
\Gamma(E)=\frac{\Omega_{0}|E|}{8 v_{F}^{2}} \sum_{\alpha= \pm}\left(V_{2}-\alpha V_{1}\right)^{2}\left[\Theta(-\alpha E)+\Theta\left(\alpha E-t_{\perp}\right)\right] .
$$

The relative contributions of the eight BLG bands to $\Gamma(E)$ depend on the ratio of tunneling amplitudes $V_{1} / V_{2}$. Figure 1 (c) sketches the variation of $\Gamma(E)$ for $V_{1}=V_{2}$. The coupling of the impurity to BLG breaks particle-hole symmetry in general, so that the symmetry of $\rho(E)$ under $E \rightarrow-E$ is broken in $\Gamma(E)$, which has a gap with a jump onset at its lower edge $\left(E=-t_{\perp}\right)$ and a linear-in-energy onset at the upper edge $(E=0)$. The gap in the hybridization function can be traced back to the symmetries of the band states under inversion through the BLG plane [31]. By analogy with the behavior a magnetic impurity in a host with a hard gap [33-36] or a power-law pseudogap [2-6], one expects this form of $\Gamma(E)$ to produce a free-moment $(\mathrm{FM})$ phase spanning the parameter range $-t_{\perp} \lesssim$ $\mu \lesssim 0,-U \lesssim \epsilon_{d} \lesssim 0$, surrounded by a strong-coupling (SC) phase in which the impurity moment is fully quenched at temperature $T=0$.

For $V_{1}=-V_{2}$, the hybridization in Fig. 1(c) must be reflected about $E=0$, but otherwise the physics is the same as for $V_{1}=V_{2}$. In cases $\left|V_{1}\right| \neq\left|V_{2}\right|$ that may arise if the impurity sits closer to one graphene layer than the other, Eq. (3) shows that $\Gamma(E)=0$ only at $E=0$. Nonetheless, if $V_{1}$ and $V_{2}$ have similar magnitudes, there will be a range of chemical potentials just above or below $\mu=0$ within which $\Gamma(\mu)$ is so small that any Kondo screening takes place below experimentally accessible temperatures, and the measured properties will be indistinguishable from those for $\left|V_{1}\right|=\left|V_{2}\right|$.

To substantiate the picture outlined above, we have studied the case $V_{1}=V_{2}$ using the numerical renormalization-group (NRG) [37], a nonperturbative method that allows $H_{A}$ to be diagonalized iteratively to obtain the low-lying many-body states, which can be used to calculate the impurity occupancy $\left\langle n_{d}\right\rangle$, its contribution to the static magnetic susceptibility $\chi_{\text {imp }}(T)$, and the impurity spectral function $A_{d}(\omega, T)$. We adopt units where $\hbar=k_{B}=g \mu_{B}=D=1$. All results shown are for $U=0.1$ and $V_{1}=V_{2}=0.21$, calculated with an NRG discretization parameter $\Lambda=2.5$ and retaining 2000 many-body states (each one representing a degenerate spin multiplet) after each iteration.

We first consider the global phase diagram. Figure 2 maps the $T=0$ impurity occupancy on the $\mu-\epsilon_{d}$ plane. There are

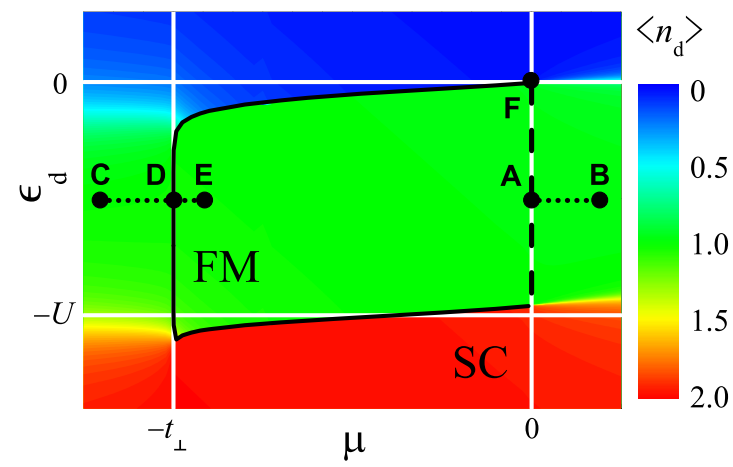

FIG. 2. (Color online) Ground-state impurity occupancy $\left\langle n_{d}\right\rangle$ on the $\mu-\epsilon_{d}$ plane for $U=0.1$ and $V_{1}=V_{2}=0.21$. Thick black lines demarcate free-moment (FM) and strong-coupling (SC) phases. The occupancy varies continuously across a line of Kosterlitz-Thouless QPTs at $\mu=0$ (dashed). Elsewhere around the phase boundary, $\left\langle n_{d}\right\rangle$ jumps across a first-order line (solid). White lines at $\mu=0,-t_{\perp}$ delimit the hybridization gap; that at $\epsilon_{d}=0(-U)$ shows where $\left\langle n_{d}\right\rangle$ would jump from 0 to 1 ( 1 to 2 ) for $V_{1}=V_{2}=0$. A through $\mathrm{F}$ schematically represent end points of paths discussed in the text. 
two phases, within each of which $\left\langle n_{d}\right\rangle$ varies smoothly under change of $\mu$ and/or $\epsilon_{d}$. An FM phase spans a contiguous region (bounded by thick lines in Fig. 2) that largely coincides with the one (inside white lines) in which $\Gamma(\mu)=0$ and the impurity level would be singly occupied in the atomic limit $V_{1}=V_{2}=0$. Throughout most of the FM phase, $0.9<\left\langle n_{d}\right\rangle<$ 1.1 , although strong departures from this range occur in the lower-left and upper-right corners. The rest of the plane is taken up by an SC phase. Around three sides (solid line) $\left\langle n_{d}\right\rangle$ jumps on crossing the phase boundary, with $\left|\Delta\left\langle n_{d}\right\rangle\right|$ exceeding 0.9 along most of the top and bottom sides but generally being smaller than 0.01 along a near-vertical section at $\mu \simeq-t_{\perp}$. By contrast, the occupancy varies smoothly across the boundary at $\mu=0$ (dashed line), where the existence of a QPT is seen in properties other than $\left\langle n_{d}\right\rangle$. The SC (green) region for $\mu<-t_{\perp}$ and $\mu>0$ would result in strong Kondo signatures in STM experiments.

We now examine the magnetic susceptibility along various paths crossing the phase boundary, beginning near the right edge. Figure 3(a) shows $T \chi_{\text {imp }}$ vs $T$ for $\epsilon_{d}=-U / 2$ and a set of $\mu$ values rising from 0 (shown schematically as path

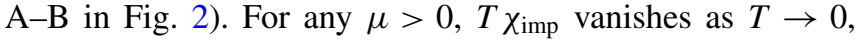
signaling the complete screening of the impurity magnetic moment at an SC fixed point. The susceptibility follows a scaling form $T \chi_{\text {imp }}(T)=f\left(T / T_{K}\right)$ for $T \lesssim T_{K}$ defined via $T_{K} \chi_{\text {imp }}\left(T_{K}\right)=0.0701$, and satisfies the standard relation $\chi_{\text {imp }}(0)=0.103 / T_{K}$ [38]. The Kondo temperature [circles in Fig. 1(d)] is well-captured by the Haldane formula [39] $T_{K}=\sqrt{U \Gamma / 2} \exp \left[\pi \epsilon_{d}\left(U+\epsilon_{d}\right) / 2 U \Gamma\right]$ [line in Fig. 1(d)] for a constant hybridization function given by $\Gamma \equiv \Gamma(\mu)$. The impurity spectral function (not shown) exhibits a Kondo resonance width proportional to $T_{K}$, and Hubbard bands near $\omega= \pm U / 2$. These properties, all characteristic of a conventional Kondo effect [38], indicate that in this range of

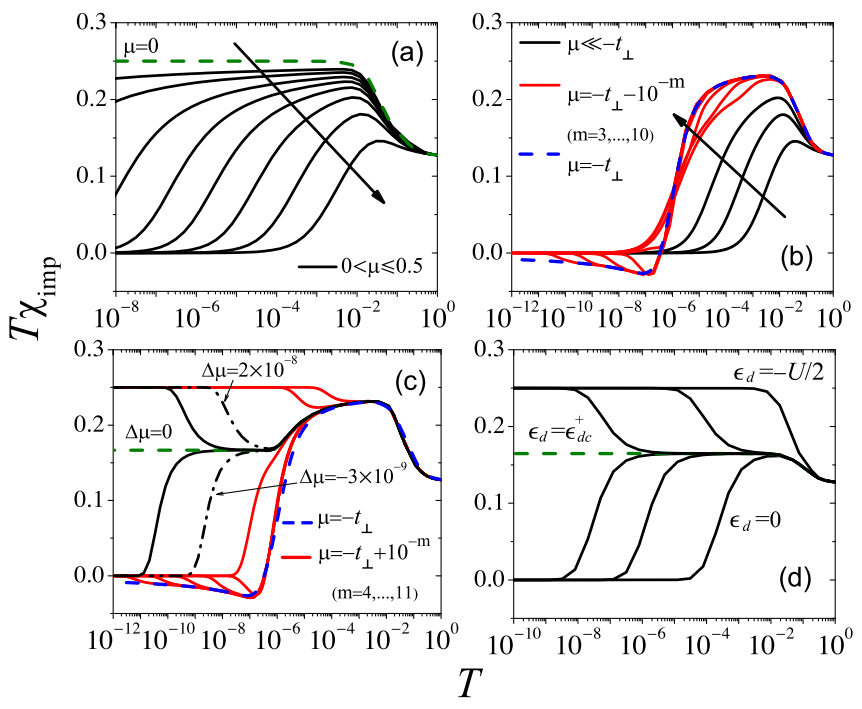

FIG. 3. (Color online) (a)-(c) Impurity susceptibility $T \chi_{\text {imp }}(T)$ for $\epsilon_{d}=-U / 2$ and different values of $\mu$ (increasing in the direction of the arrows) in the ranges (a) $0 \leqslant \mu \leqslant 0.5$, (b) $\mu \leqslant-t_{\perp}$, and (c) $-t_{\perp} \leqslant \mu<-t_{\perp}+10^{-4}$. In (c), $\Delta \mu=\mu-\mu_{c}^{-}$with $\mu_{c}^{-} \simeq-t_{\perp}+$ $1.16 \times 10^{-6}$. (d) $T \chi_{\text {imp }}(T)$ for $\mu=0$ and different values of $\epsilon_{d}$ between $-U / 2$ and 0 , with $\epsilon_{d c}^{+} \simeq-3.22 \times 10^{-4}$. Notice $t_{\perp}=0.12$. chemical potentials the variation of $\Gamma(E)$ away from $E=\mu$ has negligible effect on the Kondo physics.

As $\mu$ approaches zero from above, $T_{K}$ decreases rapidly, exhibiting an exponential sensitivity to the vanishing of $\Gamma(\mu)$, as expected from Haldane's formula. However, for $\mu=0$ (point A in Fig. 2), $T \chi_{\text {imp }}$ does not drop toward zero as $T \rightarrow 0$, but rather rises to the value $1 / 4$ produced by a free-spin- $\frac{1}{2}$ moment. For $\mu<0, T \chi_{\text {imp }}$ rises above the $\mu=0$ curve for intermediate $T$ before approaching the asymptote of $1 / 4$ (not shown). This flow to the FM fixed point has no characteristic temperature scale analogous to $T_{K}$. The impurity spectral function shows no features on scales $|\omega| \ll U / 2$. These are all properties of a Kosterlitz-Thouless (KT) QPT (similar to that found in the conventional Anderson model in the limit of vanishing hybridization), an interpretation consistent with the smooth evolution of $\left\langle n_{d}\right\rangle$ across the phase boundary. Calculations for other values of $\epsilon_{d}$ provide evidence for a line of KT fixed points at $\mu_{c}^{+}\left(\epsilon_{d}\right)=0$.

Moving to the left edge of the FM phase, we next consider $\epsilon_{d}=-U / 2$ and various $\mu$ values spanning $\mu=-t_{\perp}$ (shown schematically as path C-E in Fig. 2). Figure 3(b) shows that the $T \rightarrow 0$ behavior of $T \chi_{\text {imp }}(T)$ remains conventional for $\mu \lesssim$ $-t_{\perp}-10^{-5} \cdot T_{K}$ as defined by $T_{K} \chi_{\text {imp }}\left(T_{K}\right)=0.0701$ is in good agreement with Haldane's formula [Fig. 1(d)]. By contrast, for $\left|\mu+t_{\perp}\right| \lesssim 10^{-7}$ [Figs. 3(b) and 3(c)], over which range $T_{K}$ is almost constant, $\chi_{\text {imp }}$ changes sign at $T \simeq T_{K} / 2$ and then approaches zero from below. Such a sign change, seen in other systems with strong variation of the hybridization near the chemical potential [40-42] but generally without associated QPTs, arises from the discontinuity in $\Gamma(E)$ at $E=-t_{\perp}$ [31].

Figure 3(c) shows that upon a small increase in $\mu$ further above $-t_{\perp}, T \chi_{\text {imp }}$ reverts to approaching zero from above, but exhibits a scaling $T \chi_{\text {imp }}\left(T \ll T_{K}\right)=f\left(T / T_{K}\right)$ with a different $f$ than in the conventional Kondo regimes $\mu>0$ and $\mu \lesssim-t_{\perp}-10^{-5}$. Moreover, $T \chi_{\text {imp }}$ remains on a plateau at $1 / 6$ down to ever-lower temperatures as $\mu$ increases toward $\mu_{c}^{-}\left(\epsilon_{d}=-U / 2\right) \simeq-t_{\perp}+1.16 \times 10^{-6}$, where $T \chi_{\text {imp }}=1 / 6$ persists to $T=0$. For $\mu>\mu_{c}^{-}, T \chi_{\text {imp }}$ instead rises from the plateau to reach its free-spin- $\frac{1}{2}$ value $T \chi_{\text {imp }}=1 / 4$ as $T \rightarrow 0$. In this part of the FM phase, one may also define a crossover temperature $T_{X}$ via the criterion $T_{X} \chi_{\text {imp }}\left(T_{X}\right)=1 / 5$ (say). $T_{K}$ in the SC phase and $T_{X}$ in the FM phase both vanish linearly with $\mu-\mu_{c}^{-}$. These properties and the jump in $\left\langle n_{d}\right\rangle$ noted above point to a first-order QPT arising from the crossing of FM doublet and SC singlet ground states. Essentially the same behaviors are seen in the Anderson model with a power-law pseudogap described by a superlinear energy exponent $[4,6]$. Similar behavior to that for $\epsilon_{d}=$ $-U / 2$ occurs elsewhere along the left edge of the FM phase with the value of $\mu_{c}^{-}+t_{\perp}$ varying with $\epsilon_{d}$ but remaining small and positive.

Moving round to the top and bottom portions of the phase boundary, one no longer finds sign changes in $\chi_{\mathrm{imp}}$, but indications of a level-crossing QPT extend all the way to the corners at $\mu=0$. Figure 3(d) illustrates $T \chi_{\text {imp }}(T)$ for $\mu=0$ and different level energies in the range $0 \geqslant \epsilon_{d} \geqslant-U / 2=$ -0.05 (path A-F in Fig. 2), showing that $T \chi_{\text {imp }}=1 / 6$ persists to $T=0$ at $\epsilon_{d c}^{+}(\mu=0) \simeq-3.22 \times 10^{-4}$, representing the upper end of the first-order line. A similar QPT (not shown) anchors the lower end at $\epsilon_{d c}^{-}(\mu=0) \simeq-0.0959$. 

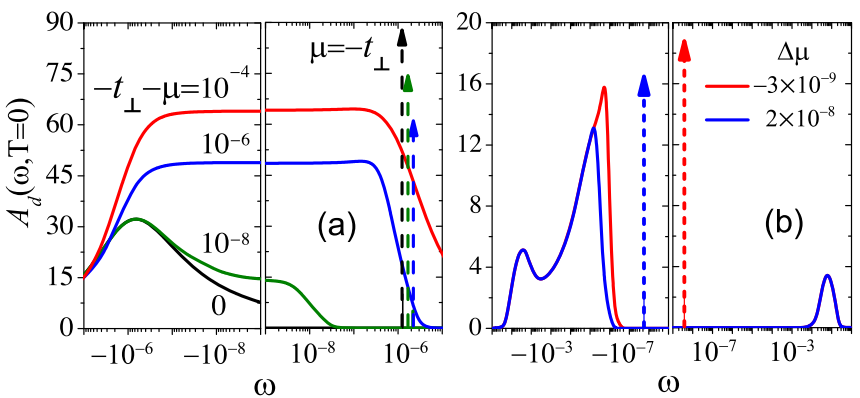

FIG. 4. (Color online) Impurity spectral function $A_{d}(\omega, T=0)$ for $\epsilon_{d}=-U / 2$ and (a) four values of $\mu$ just below $-t_{\perp}$, and (b) two values of $\mu$ straddling $\mu_{c}^{-}$. Dashed lines denote poles inside the hybridization gap, corresponding to singlet bound states.

Finally, we turn to the behavior of the $T=0$ impurity spectral function near the left edge of the FM phase, as illustrated for $\epsilon_{d}=-U / 2$ in Fig. 4 (again, path C-E in Fig. 2). Well into the Kondo regime, for $\mu+t_{\perp} \ll-T_{K}$ [see, e.g., the curve for $\mu=-t_{\perp}-10^{-4}$ in Fig. 4(a)], the only low-energy spectral feature is a Kondo resonance of width $T_{K}$ centered on $\omega=0$. As $\mu$ gets closer to $-t_{\perp}, T_{K}$ remains almost constant but the Kondo peak loses its spectral weight at frequencies $\omega>-t_{\perp}-\mu$ lying inside the hybridization gap, leaving a weaker virtual bound state resonance centered at $\omega \simeq-T_{K}$. Simultaneously, a pole appears in $A_{d}(\omega, 0)$ at a frequency $\omega_{b} \simeq T_{K}$ inside the gap. This pole is associated with a bound state produced by potential scattering from the impurity, a feature already present in the noninteracting limit $U=0$ [43] (although rescaled for $U>0$ ).

Once $\mu$ exceeds $-t_{\perp}$, the hybridization gap pushes the lowfrequency continuum portion of $A_{d}(\omega, T=0)$ into the range $\omega<-t_{\perp}-\mu$, as illustrated in Fig. 4(b) for $\Delta \mu=\mu-\mu_{c}^{-}=$ $-3 \times 10^{-9}$ and $2 \times 10^{-8}$ [values of $\mu$ corresponding to dashed lines in Fig. 3(c)]. Just as for $\mu \lesssim-t_{\perp}$, a bound state appears at a frequency $\omega_{b} \simeq T_{K}$. As $\mu$ increases through $\mu_{c}^{-}$, the bound state passes smoothly through $\omega=0$ to take up a negative frequency $\omega_{b} \simeq-T_{X}$ in the FM phase [see Fig. 4(b)], and switches character from a spin-singlet to a doublet. Further increase of $\mu$ eventually causes the bound state to merge into the continuum once $T_{X} \gtrsim t_{\perp}+\mu$.

The preceding results suggest that for $\mu$ sufficiently close to the hybridization edge at $-t_{\perp}$, scanning tunneling microscopy should detect a very sharp resonance close to the Fermi energy. Although the bound state giving rise to this resonance is of single-particle character, it may be difficult to distinguish from the many-body Kondo resonance that occurs deeper into the SC phase.

This rich and complex behavior could be probed in experimentally accessible systems such as BLG intercalated with nonmagnetic atoms (like $\mathrm{Li}$ [44], which functions as electron dopant) or molecules, together with a low concentration of magnetic impurities. A change in $\mu$ could be achieved through gating as well, in a way that does not substantially modify the original band structure [45].

In summary, we have presented nonperturbative solutions of an Anderson model describing a magnetic impurity intercalated in bilayer graphene (BLG). In the high-symmetry impurity configuration considered, tunneling interference effects combine with the BLG density of states to impart a strong energy dependence to the impurity-host hybridization function. As a consequence, the system exhibits both a Kondo-screened phase and a non-Kondo phase in which an unquenched impurity moment survives to absolute zero. The phase boundary is marked by lines of quantum phase transitions, some of which are first order while the others are of Kosterlitz-Thouless type. For chemical potentials that lie close to a jump onset in the hybridization function, single-particle bound states may give rise to signatures in scanning tunneling microscopy very similar to those produced by the many-body Kondo resonance.

This work was supported under NSF Materials World Network Grants No. DMR-1107814 (Florida) and No. DMR1108285 (Ohio), as well as by NSF-PIRE Grant No. 0730257. D.M., N.S., and S.E.U. acknowledge the hospitality of the Dahlem Center and support from the A. von Humboldt Foundation.
[1] A. C. Hewson, The Kondo Problem to Heavy Fermions (Cambridge University Press, Cambridge, 1997).

[2] D. Withoff and E. Fradkin, Phys. Rev. Lett. 64, 1835 (1990).

[3] R. Bulla, T. Pruschke, and A. C. Hewson, J. Phys.: Condens. Matter 9, 10463 (1997).

[4] C. Gonzalez-Buxton and K. Ingersent, Phys. Rev. B 57, 14254 (1998).

[5] M. T. Glossop and D. E. Logan, J. Phys.: Condens. Matter 15, 7519 (2003).

[6] L. Fritz and M. Vojta, Phys. Rev. B 70, 214427 (2004).

[7] M. Vojta and R. Bulla, Phys. Rev. B 65, 014511 (2001).

[8] K. S. Novoselov, D. Jiang, T. Booth, V. V. Khotkevich, S. M. Morozov, and A. K. Geim, Proc. Natl. Acad. Sci. USA 102, 10451 (2005).

[9] A. H. Castro Neto, F. Guinea, N. M. R. Peres, K. S. Novoselov, and A. K. Geim, Rev. Mod. Phys. 81, 109 (2009).

[10] M. Hentschel and F. Guinea, Phys. Rev. B 76, 115407 (2007).
[11] K. Sengupta and G. Baskaran, Phys. Rev. B 77, 045417 (2008).

[12] P. S. Cornaglia, G. Usaj, and C. A. Balseiro, Phys. Rev. Lett. 102, 046801 (2009).

[13] T. O. Wehling, A. V. Balatsky, M. I. Katsnelson, A. I. Lichtenstein, and A. Rosch, Phys. Rev. B 81, 115427 (2010).

[14] Z.-G. Zhu, K.-H. Ding, and J. Berakdar, Europhys. Lett. 90, 67001 (2010).

[15] M. Vojta, L. Fritz, and R. Bulla, Europhys. Lett. 90, 27006 (2010).

[16] D. Jacob and G. Kotliar, Phys. Rev. B 82, 085423 (2010).

[17] S.-P. Chao and V. Aji, Phys. Rev. B 83, 165449 (2011).

[18] B. Uchoa, T. G. Rappoport, and A. H. Castro Neto, Phys. Rev. Lett. 106, 016801 (2011).

[19] T. Kanao, H. Matsuura, and M. Ogata, J. Phys. Soc. Jpn. 81, 063709 (2012).

[20] M. A. Cazalilla, A. Iucci, F. Guinea, and A. H. Castro Neto, arXiv:1207.3135. 
[21] M. Kharitonov and G. Kotliar, Phys. Rev. B 88, 201103(R) (2013).

[22] V. W. Brar, R. Decker, H.-M. Solowan, Y. Wang, L. Maserati, K. T. Chan, H. Lee, C. O. Girit, A. Zettl, S. G. Louie, M. Cohen, and M. Crommie, Nat. Phys. 7, 43 (2010).

[23] L. S. Mattos, C. R. Moon, M. W. Sprinkle, C. Berger, K. Sengupta, A. V. Balatsky, W. A. de Heer, and H. C. Manoharan (unpublished).

[24] J.-H. Chen, L. Li, W. G. Cullen, E. D. Williams, and M. S. Fuhrer, Nat. Phys. 7, 535 (2011).

[25] X. Hong, K. Zou, B. Wang, S.-H. Cheng, and J. Zhu, Phys. Rev. Lett. 108, 226602 (2012).

[26] L. Fritz and M. Vojta, Rep. Prog. Phys. 76, 032501 (2013).

[27] E. V. Castro, K. S. Novoselov, S. V. Morozov, N. M. R. Peres, J. M. B. Lopes dos Santos, J. Nilsson, F. Guinea, A. K. Geim, and A. H. Castro Neto, Phys. Rev. Lett. 99, 216802 (2007).

[28] K.-H. Ding, Z.-G. Zhu, and J. Berakdar, J. Phys.: Condens. Matter 21, 182002 (2009).

[29] M. Killi, D. Heidarian, and A. Paramekanti, New J. Phys. 13, 053043 (2011).

[30] E. McCann and V. I. Fa'lko, Phys. Rev. Lett. 96, 086805 (2006).

[31] See Supplemental Material at http://link.aps.org/supplemental/ 10.1103/PhysRevB.89.081101 for details.
[32] T. P. Kaloni, M. U. Kahaly, and U. Schwingenschlögl, J. Mater. Chem. 21, 18681 (2011).

[33] K. Chen and C. Jayaprakash, Phys. Rev. B 57, 5225 (1998).

[34] M. R. Galpin and D. E. Logan, Phys. Rev. B 77, 195108 (2008).

[35] M. R. Galpin and D. E. Logan, Eur. Phys. J. B 62, 129 (2008).

[36] C. P. Moca and A. Roman, Phys. Rev. B 81, 235106 (2010).

[37] R. Bulla, T. A. Costi, and T. Pruschke, Rev. Mod. Phys. 80, 395 (2008).

[38] K. G. Wilson, Rev. Mod. Phys. 47, 773 (1975).

[39] F. D. M. Haldane, Phys. Rev. Lett. 40, 416 (1978).

[40] W. Hofstetter and S. Kehrein, Phys. Rev. B 59, R12732 (1999).

[41] L. G. G. V. Dias da Silva, N. P. Sandler, K. Ingersent, and S. E. Ulloa, Phys. Rev. Lett. 97, 096603 (2006).

[42] A. K. Zhuravlev and V. Y. Irkhin, Phys. Rev. B 84, 245111 (2011).

[43] A. V. Balatsky, I. Vekhter, and J.-X. Zhu, Rev. Mod. Phys. 78, 373 (2006).

[44] T. Kaloni, Y. Cheng, M. U. Kahaly, and U. Schwingenschlögl, Chem. Phys. Lett. 534, 29 (2012).

[45] A. B. Kuzmenko, E. van Heumen, D. van der Marel, P. Lerch, P. Blake, K. S. Novoselov, and A. K. Geim, Phys. Rev. B 79, 115441 (2009). 